\title{
Sport as a Treatment Aimed to the Recovery in Severe Psychiatric Disorders: Health, Functioning and Adjustment
}

\section{Giorgio Corretti ${ }^{*}$, Roberta Guerra ${ }^{1}$, Christian Martini ${ }^{2}$, Fabrizio Lazzerini ${ }^{1}$ and Alfredo Sbrana $^{3}$}

${ }^{1}$ UFSMA ASL Toscana Nord Ovest Zona Valdera, Italy

${ }^{2}$ Paim Coop, Italy

${ }^{3} S P D C$ Pisa ASL Toscana Nord Ovest, Italy

\section{Introduction}

According to the Council of Europe, Sport could be defined as "all forms of physical activity which, through casual or organized participation, aim at expressing or improving physical fitness and mental well-being, forming social relationships or obtaining results in competition at all levels" (752 Meeting of the Ministers' Deputies Meetings 2002 - 2001/16 May 2001) [1].

Physical and social health of people with a mental disorder is worse compared with general population [2]. Symptoms such as anhedonia, hyploergia, social avoidance, blunted affect, poor rapport, often contribute to a poorer health condition. People suffering from a severe mental disorder are more likely to have a sedentary lifestyle [3]. Psychopharmacology side effects, such as a reduction in motion, metabolic effect, obesity, play a role in worsening the general health condition.

A growing body of literature has examined how sport is linked to an increase in individual confidence, self-efficacy, social confidence and sense of belonging [4].

Also, sport may reduce BMI and promote a higher level of physical health $[5,6]$. Enjoyment and amusement are very important parts of sport activities $[7,8]$ and that could help patient adherence. More recent evidence [9] have shown a positive effect of sport on schizophrenia symptoms. However, there is a lack of evidence about the use of sport as a tool for an heterogeneous group of disorders.

The aim of our study was to assess the effectiveness of a rehabilitation through sport treatment (SR) on health, functioning and adjustment in a group of patients affected by Severe Psychiatric Disorders (SPD), such as Schizophrenia (S), Bipolar Disorder (BD), Severe Per-

sonality Disorders (PD), Autistic Spectrum (A).

\section{Material and Method}

\section{Participants}

Fifty-three patients with SPD (86.5\% male and 13.5\% female) participated in this study. Patients were recruited through the psychiatric medical unit were the study was conducted (Valdera Unit of Mental Health). Their mean age was 32.42 (SD 10.93). Schizophrenia was the prevalent diagnosis in 32.8\%, while Bipolar Disorder was $36.5 \%$, Severe Personality Disorders were $11.5 \%$, and Autistic Spectrum was 19.2\%.

\section{Materials}

We focused our observation on three main areas: the point of view of the mental health physician, the point of view of the patient and the level of community life.

The first area was identified by the extent of the utilization of the Mental Health Care Services which was measured by checking the electronic clinical chart for each person participating at the study. By doing so we obtained the following parameters: the number of ad-

\footnotetext{
*Corresponding author: Giorgio Corretti, UFSMA ASL Toscana Nord Ovest Zona Valdera, Unit of Mental Health, Via Roma 157, 56025 Pontedera (PI), Tuscany, Italy, Tel: +393393-001-211
}

Accepted: September 27, 2018;

Published online: September 29, 2018

Citation: Corretti G, Guerra R, Martini C, et al. (2018) Sport as a Treatment Aimed to the Recovery in Severe Psychiatric Disorders: Health, Functioning and Adjustment. J Mood Disord Ther 1(1):29-32

Copyright: (c) 2018 Corretti G, et al. This is an open-access article distributed under the terms of the Creative Commons Attribution License, which permits unrestricted use, distribution, and reproduction in any medium, provided the original author and source are credited. 
Citation: Corretti G, Guerra R, Martini C, et al. (2018) Sport as a Treatment Aimed to the Recovery in Severe Psychiatric Disorders: Health, Functioning and Adjustment. J Mood Disord Ther 1(1):29-32

mittance per month to the Unit of Mental Health for Hospitalization $(\mathrm{H})$, the global number of Psychiatric Emergency per month (PE), the number of Psychiatric Evaluation per month (PV), and Other Psychiatric Performance, such as informal conversation, interview, life support, etc. (OPP) per month. We refers at those observations as "health variables".

The second area consisted in the level of adaptation, measured by the Longitudinal Interval Follow-up Evaluation scale (LIFE) [10] which is a semi-structured interview wildly use to assess the course of the severity of the disorder [11].

The third area was about the assessment of the level of functioning, which was measured with the The Global Assessment of Functioning scale (GAF) [12]. The GAF represent the judgment of the physician about a patient level of functioning [13].

Both Scale were administered by a trained clinician and all patients were evaluated at baseline, after 12 and 24 months.

We have collected information about diagnosis by checking each patient medical chart. Since all the patients participating at the study were in charge to the Unit for a long-time diagnosis were previously made by expert psychiatrist based on the DSM-IV Axis I and II Disorders.

\section{Procedures}

The SR treatment applied was a 24 months' program with different sports such as football, basketball, fencing, archery, rugby, alone or in association with each other, depending on the characteristics of the patient, using an intent-to-treat strategy. The SR treatment was added to the already existing treatment (psychopharmacology and/or psychotherapy). The SR program was conducted from one to multiple sessions per week, each session lasted 90 minutes. Sessions were conducted by a professional coach for each sport and a Mental Health Technician who played together with the patients.

Patients were included in the study after a confirmation of their participation: they started SR after 3 weeks. If they interrupted later than the first 3 weeks, they were signed "drop out".

Two factors influenced each session of the program: patient - physician relationship and amusement. The quality of the patient-physician relationship has an important role in in the effectiveness of the treatment [14]. Also, amusement and enjoyment represent crucial factors that help to maintain attention, interest and comprehension to an effective treatment.

Data were entered and analyzed using the Statistical Package for Social Science (SPSS) version 18. Descriptive statistics were used to compute mean and standard deviation of scores.

A t-test for paired sample was run to determine whether health variables, adaptation and functioning were different after 12 and 24 months of treatment.

\section{Results}

There was a significant difference in the mean of scores for the number of hospitalizations $(\mathrm{H})$ per month which

Table 1: $t$-test results for the Utilization of Mental Health Care Services: "Health variables".

\begin{tabular}{|l|l|l|l|l|l|l|}
\hline & Baseline & \multicolumn{2}{l|}{ After 12 months } & \multicolumn{3}{|c|}{ After 24 months } \\
\hline & $\mathbf{M}$ & SD & M & t & M & t \\
\hline H & 0.091 & 0.251 & $0.003^{*}$ & 2.5 & 0.001 & $2.41^{*}$ \\
\hline PE & 0.135 & 0.281 & $0.024^{* *}$ & 3.03 & 0.027 & $2.90^{* *}$ \\
\hline PV & 0.992 & 0.928 & $0.562^{* *}$ & 3.07 & 0.499 & $3.74^{* *}$ \\
\hline OPP & 5.716 & 7.338 & 4.540 & 1.46 & 5.123 & 0.65 \\
\hline
\end{tabular}

$\mathrm{H}$ : Number of Hospitalization per month; PE: Global number of Psychiatric Emergency per month; PV: Number of Psychiatric Evaluation per month; OPP: Other Psychiatric Performance per month.

${ }^{*} p<0.05 ;{ }^{* *} p<0.01$.

Table 2: $t$-test results for adjustment and functioning levels.

\begin{tabular}{|l|l|l|l|l|l|l|}
\hline & \multicolumn{2}{l}{ Baseline } & \multicolumn{2}{l|}{ After 12 months } & \multicolumn{3}{l|}{ After 24 months } \\
\hline & M & SD & M & t & M & t \\
\hline Work adjustment & 5.73 & 0.68 & 5.23 & $4.23^{* *}$ & 5.10 & 4.61 \\
\hline Homeworks & 5.44 & 0.94 & 5.12 & $4.03^{* *}$ & 4.85 & 4.82 \\
\hline Familiar relationship & 4.73 & 0.73 & 4.33 & $4.59^{* *}$ & 4.10 & 5.44 \\
\hline Friendship & 5.12 & 0.75 & 4.21 & $7.50^{* *}$ & 3.90 & 8.94 \\
\hline Enjoyment & 4.67 & 0.76 & 3.87 & $7.85^{* *}$ & 3.56 & 9.16 \\
\hline Global satisfaction & 4.83 & 0.77 & 3.98 & $6.99^{* *}$ & 3.60 & 8.68 \\
\hline Global adaptation & 4.94 & 0.73 & 4.25 & $6.19^{* *}$ & 3.94 & 7.43 \\
\hline Global Function & 35.69 & 14.17 & 49.56 & $-7.50^{* *}$ & 54.96 & -8.52 \\
\hline
\end{tabular}

* $p<0.05 ;{ }^{* *} p<0.01$. 
Citation: Corretti G, Guerra R, Martini C, et al. (2018) Sport as a Treatment Aimed to the Recovery in Severe Psychiatric Disorders: Health, Functioning and Adjustment. J Mood Disord Ther 1(1):29-32

at beginning was 0.09 , after 12 months it became 0.003 and at the end of the study it was 0.009 . PE at beginning was 0.135 , it became 0.024 after 12 months and 0.027 after 24 months. PV at beginning was 0.99 , after 12 months it became 0.56 and it was 0.49 at the end of the study. OPP at baseline was 5.71, while after 12 months it became 4.54 , and after 24 months it became 5.12. (Table 1).

The t-test was associated with a statistically significant difference also for adjustment variables mean scores. The value of Work adjustment at beginning was 5.73, it became 5.23 after 12 months and 5.10 after 24 months. Home works were 5.44 and became 5.12 after 12 months and 4.85 after 24 months. The initial value of familiar relationships was 4.73 , after 12 months it was 4.33 and at the end it was 4.10. Friendship at beginning was 5.12, it reduced at 4.21 after 12 months and at 3.90 after 24 months. Enjoyment at the start was 4.67, then after 12 months was 3.87 and 3.56 after 12 months. Global satisfaction passed from 4.83 at beginning, to 3.98 after 12 months and 3.60 after 24 months. Global adaptation was 4.94 at beginning ad it was 4.25 after 12 months and 3.94 after 24 after 24 months (Table 2 and Figure 1).

Global level of function of the whole group was 35.69 at beginning, and it increased to 49.56 after 12 months and it went up to 54.96 at the end of the study (Table 2 and Figure 2).
Each difference in health variable showed a statistically significant difference except for OPP. These results suggest that using sport as a rehabilitation treatment does have an effect on health, function and adjustment. Differences in scores suggest that SR not only improves clinical outcome, but also the quality of life under many other aspects such as relationship, work and global satisfaction. Specifically, our results suggest that sport can be effective on reducing the number of utilization of the Mental Health Care Services. However, the treatment doesn't have an impact on the number of standard access to the Unit.

Only one patient dropped out after more than 3 weeks (1.8\%), 52 patients completed the study meaning that the intervention was well accepted by patients.

\section{Discussion}

Over the lasts years, there has been an exponential growth in the development of new techniques in psychiatric rehabilitation [15]. Sport and physical exercise represent a new kind of tool with a growing use in mental health interventions [16]. Recently sport is widely implemented in many rehabilitation programs. In our opinion the reasons for its success reside in the low cost of activities, the easily application to an heterogeneous groups (diagnoses, age, gender, etc) and the positive impact on health, functioning and adjustment. Also, it is well accepted by the patients.

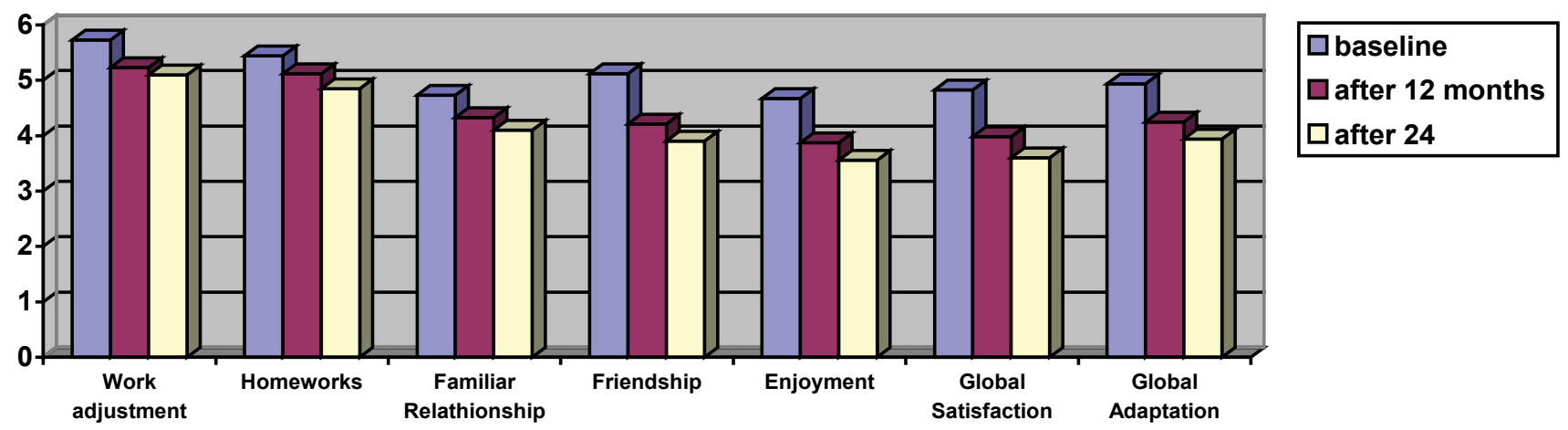

Figure 1: Comparison of mean scores for adaptation.

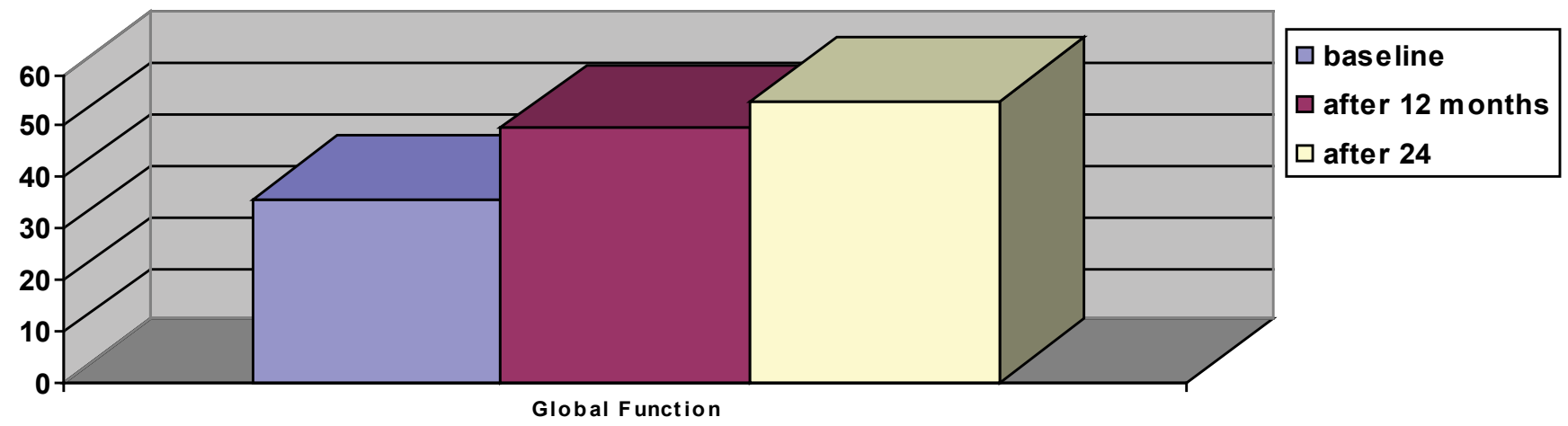

Figure 2: Comparison of mean scores for functioning. 
Citation: Corretti G, Guerra R, Martini C, et al. (2018) Sport as a Treatment Aimed to the Recovery in Severe Psychiatric Disorders: Health, Functioning and Adjustment. J Mood Disord Ther 1(1):29-32

As expected the rate of hospitalization and psychiatric emergencies was reduced after the introduction of the SR both after 12 months and 24 months. These variables are closely linked to the state of severity of the acute symptoms, meaning that there was a reduction in the number of relapses. A decrease of psychiatric emergencies reduced the unscheduled interventions with beneficial for the whole psychiatric team. The reduction in number of $\mathrm{PV}$ underline the positive course of the disorder allowing the team to focus its attention to other critical situations. OPP did not decrease during the intervention, this is not surprising since this variable is about the "standard" access to the mental health services and doesn't give information about the exacerbation of symptomatology.

SR was well-accepted by the patients for the absence of side effect, the easy access and the enjoyment during the activities. SR improves satisfaction and enjoyment, no one of our patients felt bad during activities. The abilities acquired through SR are used during patient's daily life, which led to an increase in their familiar and work, adjustment.

Our results show a relevant increase of global function both after 12 and 24 months, these findings suggest that sport can change patient inability state into a productivity condition.

Our study has several limitations: it isn't a case-control study, our data represent a comparison between variables pre and post-treatment. Our group is composed by different diagnoses, and our group isn't enough wide to describe how a diagnosis affect the response to the SR. The patients' clinical background is heterogeneous, such as the pharmacological treatment: the medication remained unchanged during the study. In addition to this we didn't consider the different cardiovascular stimulation among the physical activities, and the interactions with the patients' mental health: cardiovascular stimulation in rugby is higher than in archery.

The evidence from this study suggest that sport is an effective tool in psychiatric rehabilitation. Sport programs can increase adjustment and functioning towards a complete recovery.

\section{Acknowledgement}

Thanks to J Mammini, E Callari, F Gasperini, M Reino, C Carlotti, G Pantani, P Becattini, P Vetturi.

\section{References}

1. https://search.coe.int/cm/Pages/result_details.aspx?ObjectID=09000016804c9dbb
2. Soundy A, Faulkner G, Taylor A (2007) Exploring variability and perceptions of lifestyle physical activity among individuals with severe and enduring mental health problems: A qualitative study. JMH 16: 493-503.

3. Soundy A, Wampers M, Probst M, et al. (2013) Physical activity and sedentary behaviour in outpatients with schizophrenia: A systematic review and meta-analysis. Int J Ther Rehabil 20: 588-596.

4. Corretti G, Martini C, Greco PL, et al. (2011) Sport in psychiatric rehabilitation: A tool in pre-acute, post-acute and chronic phase. Int J Clin Med 2: 568-569.

5. Battaglia G, Alesi M, Inguglia M, et al. (2013) Soccer practice as an add-on treatment in the management of individuals with a diagnosis of schizophrenia. Neuropsychiatr Dis Treat 9: 595-603.

6. Takahashi H, Sassa T, Shibuya T, et al. (2012) Effect of sports participation on psychiatric symptoms and brain activations during sports observation in schizophrenia. Transl Psychiatry 2: e96.

7. Clark G, Goering P, Tomlinson G (1991) Challenging expectations: Client perceptions of white water canoeing. Psychiatr Rehabili J 14: 71-76.

8. Lamont E, Harris J, McDonald G, et al. (2017) Qualitative investigation of the role of collaborative football and walking football groups in mental health recovery. Mental Health and Physical Activity 12: 116-123.

9. Cerino S, Cirulli F, Chiarotti F, et al. (2011) Non-conventional psychiatric rehabilitation in schizophrenia using therapeutic riding: The FISE multicentre Pindar project. Ann Ist Super Sanità 47: 409-414.

10. Keller MB, Lavori PW, Friedman B, et al. (1987) The Longitudinal Interval Follow-up Evaluation A Comprehensive Method for Assessing Outcome in Prospective Longitudinal Studies. Arch Gen Psychiatry 44: 540-548.

11. Katharine A Phillips, Maria E Pagano, William Menard, et al. (2006) A 12-month follow-up study of the course of body dysmorphic disorder. Am J Psychiatry 163: 907-991.

12. Hall RC (1995) Global assessment of functioning: A modified scale. Psychosomatics 36: 267-275.

13. Abbo C, Okello E, Nakku J (2013) Effect of brief training on reliability and applicability of Global Assessment of functioning scale by Psychiatric clinical officers in Uganda. Afr Health Sci 13: 78-81.

14. Eveleigh RM, Muskens E, Van Ravesteijn H, et al. (2012) An overview of 19 instruments assessing the doctor-patient relationship: Different models or concepts are used. J Clin Epidemiol 65: 10-15.

15. Rössler W (2006) Psychiatric rehabilitation today: An overview. World Psychiatry 5: 151-157.

16. Zschucke E, Gaudlitz K, Ströhle A (2013) Exercise and physical activity in mental disorders: Clinical and experimental evidence. J Prev Med Public Health 46: S12-S21. 